\title{
Prospective analysis of craniofacial soccer incidents during FIFA competitions: an observational study
}

\author{
Mateus de Azevedo KINALSKI(a) iD \\ Kave COLLARES(b) \\ Marcos Britto CORREA(a)
}

(a) Universidade Federal de Pelotas - UFPel, School of Dentistry, Pelotas, RS, Brazil.

(b) Universidade de Passo Fundo, School of Dentistry, Passo Fundo, RS, Brazil.

Declaration of Interests: The authors certify that they have no commercial or associative interest that represents a conflict of interest in connection with the manuscript.

\section{Corresponding Author:}

Marcos Britto Correa

E-mail:marcos.britto@ufpel.edu.br

https://doi.org/10.1590/1807-3107bor-2020.vol34.0106

Submitted: September 20, 22019

Accepted for publication: January 20, 2020

Last revision: July 16, 2020
Abstract: The aim of this prospective epidemiological study was to evaluate the occurrence of incidents involving the craniofacial region of soccer players during three official FIFA competitions. The craniofacial incidents were identified by video analysis of all 144 matches of two FIFA World Cups (2014/2018) and the 2017 FIFA Confederations Cup. Data collection included incident type, incident severity, site affected, incident cause and referee decision. The multivariate Poisson regression model was performed to analyze the associations between covariates. A total of 238 incidents were observed in the craniofacial region (1.6 incidents/match), representing a rate of 48.5 incidents per 1000 hours. At least $80.6 \%$ of the matches presented at least one incident, and, in more than $60 \%$, the referee's decision was no foul. According to severity, $26.8 \%$ of the incidents were classified as having mild or high severity. Incidents involving lacerations or fracture presented higher severity compared with hits (IRR 3.45[95\%CI: 1.89-6.30]). Head-to-head impacts showed an incidence of severe incidents twice as high as those involving upper extremities (IRR 2.01[95\%CI:1.07-3.76]). A high number of craniofacial incidents were observed in the last FIFA competitions. Head-to-head impacts and lacerations or fractures were associated with higher incident severity.

Keywords: Prospective Studies; Soccer; Wounds and Injuries; Craniocerebral Trauma; Facial Injuries.

\section{Introduction}

Soccer is the most popular collective sport in the world, and an estimated 270 million people practice it worldwide. ${ }^{1}$ Playing soccer involves relative risk of injuries to players, owing to multiple physical interactions during matches or practice. ${ }^{2}$ In this respect, the emerging competitiveness and today's physical development of athletes may have affected the increase in soccer-related injuries, compared with the scenario prevailing in the last decades. ${ }^{3,4}$ A recent systematic review showed that the injury rate during professional soccer competitions ranged from 8.7-65.9 per 1,000h played, ${ }^{2}$ whereas the overall incidence of head injuries was 12.5 per $1,000 \mathrm{~h}$ played. ${ }^{5}$ Although the lower extremities are the body region most frequently affected by soccer incidents, ${ }^{6}$ craniofacial incidents were also associated with heavy injuries and potential risk to the physical integrity of soccer athletes during soccer games. ${ }^{78}$ 
Soccer is a contact sport and head injuries can occur during its practice. Heading the ball is one of the most common plays in a soccer match. Although there are different studies evaluating the mechanism and consequences of heading, ${ }^{9,10}$ this action remains an essential part of the game, and is a crucial play to soccer athletes during the game. In addition, craniofacial contact with body parts (especially, lower extremities, upper extremities and the head) and objects (such as the ball, ground and goal) are responsible for mild, moderate and severe traumas in soccer players. ${ }^{11}$ Although there are several studies evaluating soccer-related injuries, the specific evaluation of craniofacial injuries in professional competitions remains deficient. Noteworthily, these head incidents showed a considerable increase in a 10 -year period in Norwegian soccer competition, ${ }^{12}$ and stressed the importance of making more precise assessments of the impact of craniofacial injuries. Some authors have also highlighted that soccer injuries, including craniofacial lesions, directly impact the player not only in exercising his activities, but also and more specifically in matches, because the player must recover quickly to resume the game., ${ }^{4,8,13}$ Furthermore, it was found that soccer athletes who suffer an injury while playing soccer present a greater risk when a recurrent injury occurs. ${ }^{14,15}$ Amateur players suffering head traumas associated with mild cognitive impairment ${ }^{16}$ could experience a more impacting health hazard if exposed to an additional craniofacial injury.

Among the official soccer competitions, the World Cup organized by Fédération Internationale de Football Association (FIFA) is the most notable global sports event worldwide. Owing to the importance and visibility of FIFA competitions, more in-depth knowledge of when, why and how craniofacial injuries occur throughout these events could allow a better understanding of the mechanisms related to these incidents. Video analysis of soccer matches has been described as a helpful, simple and cost-effective tool to evaluate soccer injuries, since researchers can analyze plays repeatedly, and detect the related factors that influence these incidents more precisely. ${ }^{5,8,17}$ According to Andersen et al., ${ }^{3}$ all the head injuries that occur during a soccer competition can be identified through video analysis. Hence, the aim of the present study was to evaluate incidents involving the craniofacial region, among soccer players during the 2014 FIFA World Cup, 2017 FIFA Confederations Cup and 2018 FIFA World Cup using video analysis, by investigating factors associated with these incidents.

\section{Methodology}

The present study was reported in accordance with the statements in Strengthening the Reporting of Observational Studies in Epidemiology (STROBE). ${ }^{18}$

This prospective epidemiological study assessed incidents involving risk of potential injury and actual injury detected in the analysis of all 144 matches of the 2014 FIFA World Cup (64 matches), 2017 FIFA Confederations Cup (16 matches) and 2018 FIFA World Cup (64 matches). The matches were analyzed by videotapes made available on the internet by Globo Television Network (Brazil). Each match was recorded by more than ten cameras. Full matches were analyzed in duplicate by two independent and trained researchers (M.A.K and K.C). The data obtained were analyzed and compared after each game, and any disagreement regarding the evaluated criteria was mediated by the study coordinator (M.B.C.), who was consulted to weigh in on the specific incident. In these cases, a consensus was reached after a new observation was made of the incident by the researcher's team. The incidents or injuries identified by video analysis were classified according to several characteristics related to the incident, players and game situation.

An incident was recorded when at least one player was hit in the craniofacial region by his opponent, or when another game element (ground, ball) caused the player to fall on the field for at least 15 seconds after the incident, or else, when the player seemed to be in pain. ${ }^{8}$ The severity of the incident was classified as: low, when the player laid on the grass for at least 15 seconds after being hit, or appeared to be in pain, but did not receive any medical assistance; moderate, when the player received "first aid" off the field but continued in the game; and severe when the player was substituted during the match, after receiving medical treatment for the incident or injury. 
The bodily region affected by the incident was divided into head and face, and the face was categorized into thirds, as follows: the upper third, from the frontal bone to the upper limit of the eye orbit; the middle third, from the zygomatic arch to the upper lips; and the lower third, from the lower lips to the bottom of the mandible. Incident types were classified as a hit, laceration, and fracture. In cases when multiple types of incidents occurred, the most severe outranked the others. Since these competitions receive widespread coverage by the media, fracture diagnosis was defined by consulting the official federation website or news from main media websites, in those cases in which it could not be identified by video analysis. The search was initiated when a player left the game due to an incident or injury, and a suspected fracture was identified by video analysis. The causing agent was defined as the part of the opponent's body or the game element (field or ball) that caused the craniofacial incident. The following player positions were considered: goalkeeper, side back, center back, middle fielder and striker. The site where the incident occurred on the field was recorded and categorized into the following four zones: defense, middle field, forward and penalty areas (defense and forward). Time was assessed continuously and later categorized into sextiles (each half of the game was divided into three parts lasting 15 minutes each). In matches with extra time, a new group was included. Airplay was classified as "yes" or "no" according to the players' action and to the ball position at the moment of the incident. Referee decision was categorized into "no foul" and "foul." When at least one of the teams was eliminated, the match was recorded as a "decisive" match. Matches from qualifying rounds that did not represent a risk of elimination to any team were considered "not decisive."

The distribution of incidents involving the craniofacial region was quantified per game and per 1000 playing hours. The total hours of a played match were computed as follows: 22 players $\times 90$ or 120 minutes (in case of extra time) $=33$ or 44 hours per match. To obtain a more realistic analysis of an incident occurring because of player position, a correction coefficient was used based on a 1:4:4:2 team formation, the most common among teams.
Statistical analyses were performed using Stata 14.0 software (Stata Corporation, College Station, USA). The relative and absolute frequencies of variables of interest were estimated. A multivariate Poisson regression model with robust variance was performed to analyze the associations between incident severity and variables of interest. Owing to the low frequency of severe incidents, this category was grouped together with moderate incidents to perform the analyses. Variables with p-values $\leq 0.25$ in the crude analysis were included in the model fitting process. A backward stepwise procedure was used to include or exclude explanatory variables in the model fitting. Variables included in the final adjusted model had to present a $\mathrm{p}$-value $\leq 0.25$. The variables for the final model were considered significant if they had a p-value of $\leq$ 0.05 after adjustments. Incidence ratios were obtained and estimated with $95 \%$ confidence intervals.

\section{Results}

A total of 144 matches were analyzed, 130 of which were played in regular time (90 minutes), and 14 in extra time (120 minutes). An average 126 (52.9\%) incidents occurred in a decisive match. No game was excluded from the sample. This amount represents a total of 13,380 minutes or 223 hours of gametime played. A total of 238 incidents were observed involving the craniofacial region (1.6 incidents/match), with a rate of 48.5 incidents per 1000 hours played. At least one incident occurred in 116 matches (80.6\%). Considering each competition, the 2014 FIFA World Cup presented 1.7 incidents/match, the 2017 FIFA Confederation Cup, 2.2 incidents/match, and the 2018 FIFA World Cup, 1.5 incidents/match.

Table 1 shows the descriptive analyses of the outcome and variables of interest. Of the total incidents involving the craniofacial region, hits were the most commonly observed type of injury $(92.4 \%)$, in that the head (38.2\%) and the facial middle-third (26.9\%) were the most affected sites. Regarding severity, $26.8 \%$ of the incidents were classified as having moderate or high severity, and contact with the upper extremities (arms, elbows, and shoulders) involved the most common bodily causing agents (54.1\%). In over half of the incidents, the referee's decision was "no foul." 
The middle field was the field zone where most of the incidents occurred $(46.6 \%)$, whereas the penalty area represented $26 \%$ of the incidents. The position with the most injured players was that of the strikers (139.0 incidents/1000h played).

Table 2 shows the results of the multivariateregression model for the association between variables of interest and incident severity. The adjusted model revealed that incidents with lacerations or fracture presented higher severity than hits (3.56 [CI 95\%,1.86-6.81]). Incidents caused by the head of another player showed a twofold higher potential for being considered a severe incident than incidents caused by contact with upper extremities (IRR 1.97 [95\%CI:1.01-3.86]). The model also showed that incidents occurring in the penalty area (defense or forward) posed a greater risk of higher severity than incidents occurring outside the penalty area (IRR 1.78 [95\%CI:1.02-3.10]). The referee's decision was associated with incident severity in the crude analysis; however, this association was not retained in the adjusted model.

\section{Discussion}

To the best of our knowledge, this study was the first to evaluate craniofacial incidents in official FIFA international soccer competitions. We evaluated over 230 incidents assessed through 144 matches, and over 220 hours of gametime, totaling 48.5 incidents per 1000 playing hours. The FIFA World Cup and the FIFA Confederations Cup are the most important national soccer team competitions in the world, involving more than 700 athletes in each edition. Therefore, considering the visibility and importance of these competitions, it seems relevant to assess the craniofacial incidents by video analysis, aiming to elucidate in greater detail the mechanisms of these incidents occurring while practicing soccer. It was observed that these types of incidents occur with considerable frequency during matches, affecting players differently, according to severity of the incident, type of injury and field zone of the play.

Soccer is becoming more globalized as a sport, and the demands of its athletes are growing in the same proportion. Concurrently, plays are becoming more aggressive, thus taking a greater toll on soccer-related
Table 1. Distribution of incidents by variables of interest during FIFA World Cups of 2014/2018 and FIFA Confederation Cup of 2017 ( $n=238)$.

\begin{tabular}{|c|c|c|c|}
\hline Variables & $\begin{array}{c}\text { Number of } \\
\text { Incidents (total) }\end{array}$ & $\%$ & $\begin{array}{l}\mathrm{N} / 1,000 \mathrm{~h} \\
\text { played }\end{array}$ \\
\hline \multicolumn{4}{|l|}{ Incident severity (outcome) } \\
\hline Low & 174 & 73.1 & 33.5 \\
\hline Mild & 58 & 24.3 & 11.2 \\
\hline High & 6 & 2.5 & 1.2 \\
\hline \multicolumn{4}{|l|}{ Decisive match } \\
\hline Yes & 126 & 52.9 & 24.3 \\
\hline No & 112 & 47.1 & 22.6 \\
\hline \multicolumn{4}{|l|}{ Incident type } \\
\hline Hit & 220 & 92.4 & 42.4 \\
\hline Laceration & 16 & 6.7 & 3.1 \\
\hline Fracture & 2 & 0.8 & 0.4 \\
\hline \multicolumn{4}{|l|}{ Site of incident } \\
\hline Head & 91 & 38.2 & 17.5 \\
\hline Upper third of face & 50 & 21.0 & 9.6 \\
\hline Middle third of face & 64 & 26.9 & 12.3 \\
\hline Lower third of face & 33 & 13.9 & 6.3 \\
\hline \multicolumn{4}{|l|}{ Causing agent } \\
\hline Head & 43 & 23.0 & 8.3 \\
\hline Upper extremities & 59 & 54.1 & 11.3 \\
\hline Lower extremities & 13 & 11.9 & 2.5 \\
\hline Others (ball, ground) & 12 & 11.0 & 2.3 \\
\hline \multicolumn{4}{|l|}{ Position of injured player } \\
\hline Goalkeeper & 7 & 2.9 & $15.7^{*}$ \\
\hline Right/Left back & 23 & 9.6 & $51.5^{*}$ \\
\hline Centre back & 60 & 25.2 & $126.0^{*}$ \\
\hline Middle fielder & 86 & 36.1 & $96.4^{*}$ \\
\hline Striker & 62 & 26.0 & 139.0* \\
\hline \multicolumn{4}{|l|}{ Air play } \\
\hline Yes & 148 & 62.2 & 28.5 \\
\hline No & 90 & 37.8 & 17.3 \\
\hline \multicolumn{4}{|l|}{ Referee decision } \\
\hline No foul & 134 & 60.6 & 25.8 \\
\hline Foul & 87 & 39.4 & 16.7 \\
\hline \multicolumn{4}{|l|}{ Field zone } \\
\hline Defense zone & 65 & 27.3 & 12.5 \\
\hline Middle field zone & 111 & 46.6 & 21.4 \\
\hline Forward zone & 62 & 26.0 & 12.0 \\
\hline \multicolumn{4}{|l|}{ Time of game (sextiles) } \\
\hline First & 37 & 15.6 & 7.1 \\
\hline Second & 40 & 16.9 & 7.7 \\
\hline Third & 46 & 19.4 & 8.9 \\
\hline Fourth & 28 & 11.8 & 5.4 \\
\hline Fifth & 44 & 18.6 & 8.5 \\
\hline Sixth & 38 & 16.0 & 7.3 \\
\hline Extra time & 4 & 1.7 & 0.8 \\
\hline
\end{tabular}

*\% values consider the correction coefficient based on a $1: 4: 4: 2$ formation. 
Table 2. Crude (c) and adjusted (a) incidence risk ratio (IRR) of independent variables for craniofacial incident severity in the FIFA World Cups of 2014/2018 and the FIFA Confederations Cup of 2017. Poisson regression models. $(n=238)$.

\begin{tabular}{|c|c|c|c|c|}
\hline \multirow{2}{*}{ Variable/Category } & \multicolumn{4}{|c|}{ Craniofacial incident severity (ref=Low) } \\
\hline & $\operatorname{IRR}^{c}(95 \% \mathrm{CI})$ & p-Value & $\operatorname{IRR}^{a}(95 \% \mathrm{CI})$ & $p$-Value \\
\hline Decisive match (ref=No) & & 0.434 & & 0.332 \\
\hline Yes & $1.22(0.74-2.00)$ & & $1.29(0.76-2.17)$ & \\
\hline Incident type (ref=Hit) & & $<0.001$ & & $<0.001$ \\
\hline Laceration/Fracture & $3.42(1.89-6.18)$ & & $3.66(1.90-7.02)$ & \\
\hline Injury location (ref= Head) & & 0.571 & & 0.770 \\
\hline Face & $1.16(0.69-1.94)$ & & $1.08(0.63-1.86)$ & \\
\hline Causing agent (ref=Upper extremities) & & 0.020 & & 0.131 \\
\hline Head & $2.36(1.27-4.38)$ & & $1.95(1.01-3.77)$ & \\
\hline Lower extremities & $2.33(1.16-4.70)$ & & $2.12(0.98-4.59)$ & \\
\hline Other & $1.72(0.84-3.54)$ & & $1.41(0.66-3.01)$ & \\
\hline Player position (ref=Right/left back) & & 0.145 & & 0.330 \\
\hline Goalkeeper & $3.28(0.82-13.13)$ & & $1.58(0.35-6.98)$ & \\
\hline Center back & $1.43(0.47-4.33)$ & & $1.16(0.37-3.63)$ & \\
\hline Middle fielder & $2.00(0.70-5.69)$ & & $1.93(0.66-5.59)$ & \\
\hline Striker & $1.02(0.32-3.20)$ & & $1.07(0.33-3.44)$ & \\
\hline Referee decision (ref=Foul) & & 0.014 & & 0.132 \\
\hline No Foul & $1.98(1.15-3.42)$ & & $1.55(0.87-2.77)$ & \\
\hline Air play $(r e f=\mathrm{No})$ & & 0.985 & & 0.697 \\
\hline Yes & $0.98(0.59-1.63)$ & & $0.89(0.50-1.57)$ & \\
\hline Penalty area $(r e f=\mathrm{No})$ & & 0.009 & & 0.041 \\
\hline Yes & $1.94(1.17-3.19)$ & & $1.78(1.02-3.10)$ & \\
\hline Time of game (ref $=1^{\text {st }}$ third) & & 0.374 & & 0.358 \\
\hline $2^{\text {nd }}$ third & $0.65(0.34-1.23)$ & & $0.77(0.40-1.50)$ & \\
\hline $3^{\text {rd }}$ third & $0.93(0.53-1.63)$ & & $1.26(0.69-2.31)$ & \\
\hline-2 Log-likelihood & Empty mo & 48.0 & Final mo & 29.0 \\
\hline
\end{tabular}

injuries. Importance should be given to the occasional incidents that have the potential of causing craniofacial injuries. In fact, in over half of the incidents, referees or assistant referees fail to see a fault against the injured player, thus corroborating the belief that most of the incidents are just part of the game. In addition, the penalty area posed a higher risk of moderate or high lesions compared with other field zones, because of the intense competition for ball control occurring therein. In general, this high prevalence of craniofacial incidents in the last three official FIFA soccer competitions might be explained as resulting from natural plays during soccer matches, like ball possession challenges and jumping to gain control of the ball during matches. Moreover, incidents due to head-to-head contact presented a twofold higher prevalence than those caused by contact with upper extremities, in line with the nature of a game with a high risk of injury. Although specific injuries were not evaluated in the present study, there is a well-known risk of brain damage as a result of head injuries among soccer athletes. ${ }^{3,11,19}$ Closely related to these traumas, concussion is one of the most widely acknowledged brain injuries, defined as a complex pathophysiological process affecting the brain, ${ }^{20}$ caused by a direct blow to the head, face, neck or elsewhere on the body, with an "impulsive" force transmitted to the head. Therefore, although head impacts during soccer games are common, these injuries could pose a true risk to jeopardizing a player's health. Further investigation is warranted concerning the mechanism and impact of head contacts during soccer games.

In the three competitions evaluated, the most commonly observed type of injury was concussion, 
followed by craniofacial incidents. Although it was not included among the outcomes of medium or high severity incidents, some specific situations involving concussions, occurring during the competitions under study, should be discussed in greater detail. During a match in the 2014 FIFA World Cup, one Uruguayan player suffered a hit to the head by another player's knee, and fell unconscious on the field. After the athlete regained consciousness, he argued with his team doctor to let him return to the match, without being issued a correct concussion protocol, and refused to be replaced by another player. ${ }^{21} \mathrm{~A}$ similar situation occurred in the final match of the same World Cup games, when a German midfielder suffered a heavy blow to the head. ${ }^{22}$ After this collision, he kept playing about 14 minutes without been replaced, even though he had been knocked unconscious minutes before. In light of situations such as these, and many others that have occurred in other soccer competitions, FIFA pronounced that "the incidents at the World Cup have shown that the role of team doctors has to be reinforced in order to ensure correct management of potential cases of concussion, in the heat of the competition". ${ }^{23}$ It is known that a second concussion, or another concussion sequential to the first trauma could increase the risk of brain damage. ${ }^{24,25}$ In the present study, $22 \%$ of hits to the body required medical examination on the field, or else the player had to be replaced due to a craniofacial injury. Although the injured athlete received medical attention in some cases, few reports were made available concerning the athlete's medical condition, and/or the consequence of the incident to these athletes. Recently, the National Football League (NFL) approved concussion protocols in which an independent neurotrauma consultant worked together with a physician's team to identify, screen and diagnose a concussion more precisely. ${ }^{26}$ When an incident is diagnosed as a concussion, the athlete is forbidden to return to the field, and is advised to follow medical recommendations. Similar actions should be considered and discussed for application in soccer, aiming to reduce damage to the athlete's physical integrity.

The head and face are frequently cited as injured parts of the body during soccer games. In a recent survey, the head and face were the most affected parts, followed by ankle and hands or wrist in amateur soccer players. ${ }^{27}$ In contrast, over a 6-year period of soccer fracture evaluations, the head represented only $0.1 \%$ of the reported fractures among amateur soccer players..$^{28}$ In the present study, lacerations or fractures represented a minor part of the craniofacial incidents, results that corroborate those of Bobian et al., ${ }^{29}$ in which lacerations represented the most common diagnosis of soccer-related injuries in the craniofacial region. The high variability across the data for craniofacial injuries might be attributed to the main interest shown by researchers of soccer athletes and football managers for the legs, and for disabling injuries, such as muscle strain, sprains and contusions. Although head injuries do not impair practicing soccer permanently or completely, athletes thus injured might need a rest period to recover. In these cases, specifically when short competitions are been played, like those of the FIFA World Cup or the FIFA Confederations Cup, traumas may impact games by keeping players from playing a match, and thus adversely affect the national team directly, not to mention the athlete's health.

Video analysis of soccer matches to investigate head injuries has been described in the literature. ${ }^{3,8,17}$ This methodology allows identifying different traumas using video images to analyze the effects on injured players more precisely, especially regarding the affected site, the player's position, and other variables associated with the craniofacial incident. In the FIFA 2018 World Cup, 33 video cameras were used to broadcast the games, eight of which were super slow-motion, and four, ultra-slow-motion cameras, thus providing accurate visualization of the gameplays. Furthermore, this methodology allows the researcher to interpret the craniofacial incident more "convincingly," since it enables reviewing the play using video tools to pause, rewind and check different angles of the same play in action. Nevertheless, all the images evaluated at the 144 matches were made available by FIFA's official broadcasting company, and accessed through the internet. If there were some incident that could not be recorded during the official transmission, it was because it could not be evaluated using currently available methodology. 
It is important to emphasize that observational methodology could have posed a barrier to making a more precise identification of the consequences of craniofacial injuries to an athlete's physical integrity. This is because the video analysis was performed only during official matches, and was not included in the practices, thus precluding precise investigation of all the cases occurring in the games. In addition, detailed medical diagnoses cannot be obtained using this method, which fundamentally enables researchers to differentiate incidents from real injuries. On the other hand, this prospective design enables identifying minimal craniofacial incidents during soccer games across the competitions evaluated, regardless of the incident. This is an important factor to underscore, given that these injuries may continue to pose a risk to the soccer athletes' health. The present study also provides a useful tool to help understand sports incidents, and the mechanisms involved in craniofacial injuries. It serves as an important alert to further investigate these traumas using longitudinal methodologies, which cannot be underestimated in the task of determining the reasons that caused these lesions.

\section{Conclusion}

Craniofacial incidents were observed in the great majority of the FIFA competition matches. Head-to-head impacts and lacerations or fractures were associated with higher incident severity. Considering that facial incidents are frequently considered as a natural consequence of the sport, preventive strategies should focus on adopting the use of protective equipment and promoting fair play.

\section{Acknowledgments}

This study was financed in part by the Coordination for the Improvement of Higher Education Personnel (CAPES) Finance Code 001. The authors declare no conflict of interest.

\section{References}

1. FIFA. FIFA Big count 2006: 270 million people active in football. 2006 [cited 2019 Sep 12]. Available from: http://www.fifa.com/mm/document/fifafact?s/bcoffsurv/bigcount. statspackage_7024.pd

2. Pfirrmann D, Herbst M, Ingelfinger P, Simon P, Tug S. Analysis of injury incidences in male professional adult and elite youth soccer players: a systematic review. J Athl Train. 2016 May;51(5):410-24. https://doi.org/10.4085/1062-6050-51.6.03

3. Andersen TE, Tenga A, Engebretsen L, Bahr R. Video analysis of injuries and incidents in Norwegian professional football. Br J Sports Med. 2004 Oct;38(5):626-31. https://doi.org/10.1136/bjsm.2003.007955

4. Aoki H, O’Hata N, Kohno T, Morikawa T, Seki J. A 15-year prospective epidemiological account of acute traumatic injuries during official professional soccer league matches in Japan. Am J Sports Med. 2012 May;40(5):1006-14. https://doi.org/10.1177/0363546512438695

5. Fuller CW, Junge A, Dvorak J. A six year prospective study of the incidence and causes of head and neck injuries in international football. Br J Sports Med. 2005 Aug;39 Suppl 1:i3-9. https://doi.org/10.1136/bjsm.2005.018937

6. Waldén M, Hägglund M, Ekstrand J. UEFA Champions League study: a prospective study of injuries in professional football during the 2001-2002 season. Br J Sports Med. 2005 Aug;39(8):542-6. https://doi.org/10.1136/bjsm.2004.014571

7. Andersen TE, Arnason A, Engebretsen L, Bahr R. Mechanisms of head injuries in elite football. Br J Sports Med. 2004 Dec;38(6):690-6. https://doi.org/10.1136/bjsm.2003.009357

8. Correa MB, Knabach CB, Collares K, Hallal PC, Demarco FF. Video analysis of craniofacial soccer incidents: a prospective study. J Sci Med Sport. 2012 Jan;15(1):14-8. https://doi.org/10.1016/j.jsams.2011.07.011

9. Levitch CF, Zimmerman ME, Lubin N, Kim N, Lipton RB, Stewart WF, et al. Recent and long-term soccer heading exposure is differentially associated with neuropsychological function in amateur players. J Int Neuropsychol Soc. 2018 Feb;24(2):147-55. https://doi.org/10.1017/S1355617717000790

10. McCrory PR. Brain injury and heading in soccer. BMJ. 2003 Aug;327(7411):351-2. https://doi.org/10.1136/bmj.327.7411.351

11. Bunc G, Ravnik J, Velnar T. May heading in soccer result in traumatic brain injury? A review of literature. Med Arh. 2017 Oct;71(5):356-9. https://doi.org/10.5455/medarh.2017.71.356-359

12. Biørneboe J, Bahr R, Einar Andersen T. Video analysis of situations with a high-risk for injury in Norwegian male professional football; a comparison between 2000 and 2010. Br J Sports Med. 2014 May;48(9):774-8. https://doi.org/10.1136/bjsports-2012-091856 
- Prospective analysis of craniofacial soccer incidents during FIFA competitions: an observational study

13. Sadigursky D, Braid JA, De Lira DN, Machado BA, Carneiro RJ, Colavolpe PO. The FIFA 11+ injury prevention program for soccer players: a systematic review. BMC Sports Sci Med Rehabil. 2017 Nov;9(1):18. https://doi.org/10.1186/s13102-017-0083-z

14. Hägglund $M$, Waldén $M$, Ekstrand J. Previous injury as a risk factor for injury in elite football: a prospective study over two consecutive seasons. Br J Sports Med. 2006 Sep;40(9):767-72. https://doi.org/10.1136/bjsm.2006.026609

15. Olsen L, Scanlan A, MacKay M, Babul S, Reid D, Clark M, et al. Strategies for prevention of soccer related injuries: a systematic review. Br J Sports Med. 2004 Feb;38(1):89-94. https://doi.org/10.1136/bjsm.2002.003079

16. Matser EJ, Kessels AG, Lezak MD, Jordan BD, Troost J. Neuropsychological impairment in amateur soccer players. JAMA. 1999 Sep;282(10):971-3. https://doi.org/10.1001/jama.282.10.971

17. Andersen TE, Larsen $\varnothing$, Tenga A, Engebretsen L, Bahr R. Football incident analysis: a new video based method to describe injury mechanisms in professional football. Br J Sports Med. 2003 Jun;37(3):226-32. https://doi.org/10.1136/bjsm.37.3.226

18. Elm E, Altman DG, Egger M, Pocock SJ, Gøtzsche PC, Vandenbroucke JP. The Strengthening the Reporting of Observational Studies in Epidemiology (STROBE) statement: guidelines for reporting observational studies. Lancet. 2007 Oct;370(9596):1453-7. https://doi.org/10.1016/S0140-6736(07)61602-X

19. McCrory P, Feddermann-Demont N, Dvořák J, Cassidy JD, Mclntosh A, Vos PE, et al. What is the definition of sports-related concussion: a systematic review. Br J Sports Med. 2017 Jun;51(11):877-87. https://doi.org/10.1136/bjsports-2016-097393

20. O'Connor KL, Baker MM, Dalton SL, Dompier TP, Broglio SP, Kerr ZY. Epidemiology of sport-related concussions in high school athletes: National Athletic Treatment, Injury and Outcomes Network (NATION), 2011-2012 through 2013-2014. J Athl Train. 2017 Mar;52(3):175-85. https://doi.org/10.4085/1062-6050-52.1.15

21. FIFA accused after 'unconscious' Álvaro Pereira played on for Uruguay. The Guardian 2014 Jun 20 cited 2019 Sep 10]. Available from:

22. Germany's Christoph Kramer asked referee: 'Is this the final? The Guardian. The Guardian 2014 Jul 17 cited 2019 Sep 8]. Available from: https://www.theguardian.com/football/2014/jun/20/alvaro-pereira-uruguay-england-concussion-fifa-world-cup https://www.theguardian.com/football/2014/jul/17/christoph-kramer-germany-concussion-world-cup-final-2014

23. FIFA. FIFA's Medical Committee proposes new protocol for the management of concussion. 2014 Sep 23 cited 2019 Sep 12]. Available from: https://www.fifa.com/who-we-are/news/fifa-s-medical-committee-proposes-new-protocol-for-the-management-of-c-2443024

24. Manley G, Gardner AJ, Schneider KJ, Guskiewicz KM, Bailes J, Cantu RC, et al. A systematic review of potential long-term effects of sport-related concussion. Br J Sports Med. 2017 Jun;51(12):969-77. https://doi.org/10.1136/bjsports-2017-097791

25. McCrory P, Meeuwisse WH, Aubry M, Cantu B, Dvorák J, Echemendia RJ, et al. Consensus statement on concussion in sport: the 4th International Conference on Concussion in Sport held in Zurich, November 2012. Br J Sports Med. 2013 Apr;47(5):250-8. https://doi.org/10.1136/bjsports-2013-092313

26. NFI Play Smart Play Safe.. How The NFL is advancing player health and safety. 2017 cited 2019 Sep 8]. Available from: https://www.playsmartplaysafe.com/wp-content/uploads/2017/10/nfl_factsheet_healthandsafety_102617al.pdf

27. Kerr ZY, Pierpoint LA, Currie DW, Wasserman EB, Comstock RD. Epidemiologic comparisons of soccer-related injuries presenting to emergency departments and reported within high school and collegiate settings. Inj Epidemiol. 2017 Dec;4(1):19. https://doi.org/10.1186/s40621-017-0116-9

28. Kuczinski A, Newman JM, Piuzzi NS, Sodhi N, Doran JP, Khlopas A, et al. Trends and epidemiologic factors contributing to soccer-related fractures that presented to emergency departments in the United States. Sports Health. 2019 Jan/Feb;11(1):27-31. https://doi.org/10.1177/1941738118798629

29. Bobian MR, Hanba CJ, Svider PF, Hoijat H, Folbe AJ, Eloy JA, et al. Soccer-related facial trauma: a nationwide perspective. Ann Otol Rhinol Laryngol. 2016 Dec;125(12):992-9966. https://doi.org/10.1177/0003489416668195 